\title{
Randomized controlled trial of home-based 4-week tDCS in chronic minimally conscious state
}

\author{
Géraldine Martens, MSc ${ }^{a,}$, Nicolas Lejeune, MD ${ }^{\mathrm{a}, \mathrm{b}}$, Anthony Terrence O'Brien, MD ${ }^{\mathrm{c}}$, \\ Felipe Fregni, MD, $\mathrm{PhD}^{\mathrm{c}}$, Charlotte Martial, MSc ${ }^{\mathrm{a}}$, Sarah Wannez, MSc ${ }^{\mathrm{a}}$, \\ Steven Laureys, $\mathrm{MD}, \mathrm{PhD}^{\mathrm{a}, * *, 1}$, Aurore Thibaut, $\mathrm{PhD}^{\mathrm{a}, \mathrm{c}, 1}$

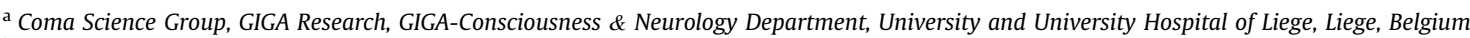 \\ ${ }^{\mathrm{b}}$ Centre Hospitalier Neurologique William Lennox, Saint-Luc University Clinics, Université Catholique de Louvain, Belgium \\ ${ }^{\mathrm{c}}$ Neuromodulation Center, Spaulding Rehabilitation Hospital, Harvard Medical School, Boston, MA, USA
}

\section{A R T I C L E I N F O}

Article history:

Received 29 December 2017

Received in revised form

26 April 2018

Accepted 28 April 2018

Available online $\mathrm{xxx}$

\section{Keywords:}

Transcranial direct current stimulation (tDCS)

Consciousness

Minimally conscious state

Traumatic brain injury

\begin{abstract}
A B S T R A C T
Background: Patients with chronic disorders of consciousness face a significant lack of treatment options. Objective: We aimed at investigating the feasibility and the behavioral effects of home-based transcranial direct current stimulation (tDCS), applied by relatives or caregivers, in chronic patients in minimally conscious state (MCS).

Methods: Each participant received, in a randomized order, 20 sessions of active and 20 sessions of sham tDCS applied over the prefrontal cortex for 4 weeks; separated by 8 weeks of washout. Level of consciousness was assessed using the Coma Recovery Scale-Revised before the first stimulation (baseline), at the end of the 20 tDCS sessions (direct effects) and 8 weeks after the end of each stimulation period (long-term effects). Reported adverse events and data relative to the adherence (i.e., amount of sessions effectively received) were collected as well.

Results: Twenty-seven patients completed the study and 22 patients received at least $80 \%$ of the stimulation sessions. All patients tolerated tDCS well, no severe adverse events were noticed after real stimulation and the overall adherence (i.e., total duration of stimulation) was good. A moderate effect size ( 0.47 and 0.53 , for modified intention to treat and per protocol analysis, respectively) was observed at the end of the 4 weeks of tDCS in favor of the active treatment.

Conclusions: We demonstrated that home-based tDCS can be used adequately outside a research facility or hospital by patients' relatives or caregivers. In addition, 4 weeks of tDCS moderately improved the recovery of signs of consciousness in chronic MCS patients.
\end{abstract}

() 2018 Elsevier Inc. All rights reserved.

\section{Introduction}

Patients who suffered from a severe brain injury and stay with a disorder of consciousness (DOC) represent a challenging population to treat since, by definition, these patients are unable to communicate, and therefore to participate in active rehabilitation programs. Currently, their treatment options are limited, especially

\footnotetext{
* Corresponding author. GIGA-Research Coma Science Group and Neurology Department, University and University Hospital of Liège, Avenue de l'Hopital, 11, Liege, Belgium.

** Corresponding author.

E-mail addresses: geraldine.martens@ulg.ac.be (G. Martens), coma@uliege.be (S. Laureys).

1 These authors share the last position.
}

in the chronic setting. Several drugs to improve the level of consciousness have been studied (e.g., zolpidem, levodopa or baclofen) [1]. However, so far, only amantadine showed significant clinical effects [2]. Recently, transcranial direct current stimulation (tDCS) showed promising results in patients in a minimally conscious state (MCS - i.e., showing fluctuating but reproducible signs of consciousness, such as response to command or visual pursuit for instance, while being unable to functionally communicate) while patients in unresponsive wakefulness syndrome (UWS - i.e., only showing cyclic eye-opening and reflexive behaviors) seem to be less responsive to that treatment. We demonstrated that a single session of tDCS, applied with the anode over the left dorsolateral prefrontal cortex (DLPFC) and the cathode over the contralateral supraorbital area, induced transient clinical improvement [4], as 
measured by the Coma Recovery Scale-Revised (CRS-R [5]). Greater effects were observed in the subgroup of MCS patients as compared to patients in UWS. Later, our group showed that applying tDCS using the same montage for five days could increase the number of responders and prolonged the duration of the effects up to one week after the end of the tDCS sessions [7]. Under a similar protocol (i.e., 5 days of tDCS with the anode positioned over the left DFLPC), Estraneo and colleagues revealed moderate improvements in consciousness in 3 patients in UWS and 3 patients in MCS (out of 13 patients with DOC) [8]. Angelakis and colleagues also identified an improvement in the CRS-R total scores following 10 days of tDCS, applied with the anode over the left DLPFC or the motor cortex, and at 12-month follow-up as well, in patients in MCS [9]. All these studies targeted the left DLPFC with the anode since this area would have an integrative role for behavior and motor control and allow better working memory and attention when stimulated [10,11]. Based on these results, tDCS seems to represent a valuable therapeutic option for MCS patients. However, currently, tDCS has to be performed by trained professionals in hospitals or research facilities, which limits its translation to clinical practice and the number of sessions; while it is known that repeated tDCS sessions are necessary to induce long-lasting effects $[12,13]$. Therefore, to address this limitation, we tested the clinical effects and the applicability of a 4-week home-based tDCS protocol in patients with chronic MCS, targeting the left DLPFC. The results may provide insight into the clinical value and application of tDCS for DOC outside of a research or hospital setting.

\section{Methods}

Standard protocol approvals, registrations, and patient consents

Written informed consents were obtained by the legal representative of each patient in accordance with the Declaration of Helsinki. This multicentric study was registered (ClinicalTrials.gov NCT02394691) and approved by the central ethics committee of the University Hospital of Liège, and by each local ethic committees as needed.

\section{Study design}

Randomized double-blind sham controlled crossover trial.

\section{Participants and eligibility criteria}

We prospectively enrolled chronic MCS patients [3], over 16 years old, more than 3 months post injury, in stable vital condition (e.g., no infection, intubation, recent hospitalization). The initial diagnosis of MCS was established based on at least five successive behavioral assessments [14]. We excluded patients with contraindication to tDCS as previously described [4], and patients who received non-invasive brain stimulation less than 3 months before their inclusion in this study. Medication and rehabilitation were kept unchanged throughout the duration of the protocol. Patients were excluded from the study if one of the following situations occurred: introduction of a new central-acting drug (e.g., antiepileptic or sedative drugs, amantadine or intrathecal baclofen); change in rehabilitation (increase or reduction of a minimum of two sessions per week); surgical intervention; infection with deterioration of patients' general condition or serious medical event (e.g., sign of pain as observed by the family or caregivers, seizure); poor adherence (i.e., patient received less than $80 \%$ of the stimulation sessions) [15].

\section{Randomization and masking}

Each patient received both active and sham tDCS stimulations in a crossover, randomized order, as presented in Fig. 1. A set of two tDCS devices (CEFALY tDCS, Cefaly Technology, Belgium) was assigned to each patient, one active and one sham. Note that only one device was given at a time to ensure that the appropriate stimulation (active or sham) was delivered. A computer-generated randomization sequence assigned in a $1: 1$ ratio the two groups: active-sham and sham-active. A third person, not involved in data collection, was responsible for the treatment allocation. Investigators, patients, and patients' relatives or caregivers were blinded to the treatment order.

\section{Procedures}

Patients were seen at home, in nursing homes or in rehabilitation facilities in Belgium, France, and Luxembourg. Caregivers (for patients in rehabilitation centers or nursing homes) or a family member (for patients at home), were trained to use the stimulation device adequately by watching a video, receiving a brochure with instructions, video-tape the placement done by an investigator and apply the electrodes themselves as a formal exam. This device was built for ease of use, with fixed parameters ( $2 \mathrm{~mA}$ during $20 \mathrm{~min}$ ), and recorded the amount and the duration of all delivered stimulations to estimate the adherence. Direct current was applied by a battery-driven constant current stimulator using saline-soaked surface sponge electrodes $(7 \times 5 \mathrm{~cm})$ with the anode (that is associated with increased cortical excitability [16]) positioned over the left DLPFC (F3 according to the 10-20 international system for EEG placement) [17] and the cathode placed over right supraorbital region. During tDCS, the current was increased during $5 \mathrm{~s}$ to $2 \mathrm{~mA}$ from the onset of stimulation, applied for $20 \mathrm{~min}$, and then ramped down to $0 \mathrm{~mA}$ for $5 \mathrm{~s}$. During sham, the intensity was ramped up to

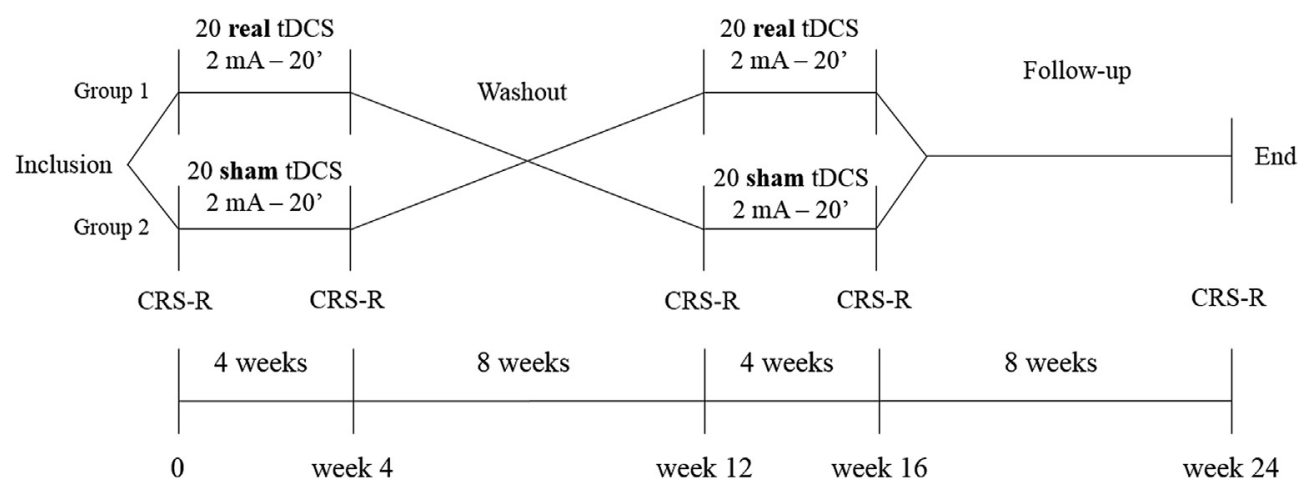

Fig. 1. Study protocol. 
$2 \mathrm{~mA}$ for $5 \mathrm{~s}$, direct current was applied for $5 \mathrm{~s}$ and then ramped down to $0 \mathrm{~mA}$ for 5 seconds. tDCS was performed daily by the same person, at the same time of the day, for 4 weeks, five times a week (20 stimulation sessions per period - the two periods were separated by 8 weeks of washout - see Fig. 1). Patients' relatives or caregivers had to report daily anything they would consider abnormal as suggested by the CRF (i.e., skin redness, skin injury, signs of pain/discomfort, epilepsy, sleepiness). During the 4 weeks stimulation period, they had to complete a detailed questionnaire (see supplementary material) including questions relative to safety and behavioral changes. Data on adherence were collected by the investigators at the end of the whole protocol, and assessments using the CRS-R were performed by trained and experienced blinded clinicians at baseline (week 0 ), week 4 (after the end of the first stimulation period) and week 12 (after 8 weeks of washout) for each period (active and sham).

\section{Outcomes}

Our primary outcome measures were: 1) safety estimation of home-based tDCS, assessed by the adverse events (AE) questionnaire; 2 ) patients' adherence to the treatment protocol, as recorded by the device; 3 ) change in the CRS-R total score after 4 weeks of tDCS.

Our secondary outcome was the change in the CRS-R total score at 8-week follow-up. As exploratory analyses, we assessed the effects of time since injury and etiology on tDCS effects and we qualitatively compared the presence of clinically relevant behaviors recovered after tDCS at the individual level.

The amount of side effects was collected based on the questionnaires and interviews with the person in charge of the stimulation.

Adherence was here defined as the total time of stimulation and was expressed as a percentage of the total duration of all the planned stimulations (i.e., 20 times $20 \mathrm{~min}$ or $6 \mathrm{~h}$ and $40 \mathrm{~min}$ ).

Patients' level of consciousness was assessed using the CRS-R which is a behavioral scale consisting of 23 hierarchically arranged items over 6 subscales assessing auditory, visual, motor, verbal, communication and arousal functions at the patient's bedside [5]. It is currently the most sensitive scale to assess consciousness and diagnose the first minimal signs of consciousness [18].

\section{Statistical analysis}

Statistical analyses were performed using Stata (StataCorp. 2013. Stata Statistical Software: Release 13. College Station, TX: StataCorp LP). Baseline characteristics between groups (activesham and sham-active) were tested for comparability using Student's t-test for continuous variables and Chi square test for dichotomous variables. We first looked at the carry-over effect with a Wilcoxon match-paired signed-rank test comparing the CRS-R total scores of the two baseline conditions (before active treatment and before sham treatment). If no carry-over effect was found, the treatment effect was then assessed using a Wilcoxon match-paired signed-rank test. The treatment effect per group at 4 weeks of treatment and at 8 weeks of follow-up was analyzed using the differences in CRS-R total score (i.e., delta), between [week $4-$ baseline] and [week 12 - baseline]. We used both modified intention to treat (MITT) [19], and per protocol (PP) [20] approaches to analyze the data. For the mITT approach, we considered data from subjects that underwent all CRS-R assessments pre- and poststimulation for both the sham and active tDCS sessions. While for the PP analysis, after collecting the adherence data from each device, we excluded subjects who did not receive at least $80 \%$ of the pre-specified number of sessions as per exclusion criteria. Results were considered significant at $\mathrm{p}<0.05$. The Cohen's d effect size was calculated from the difference in means and standard deviations between baseline and post-treatment comparing active with sham $\mathrm{tDCS}$. We then calculated the correlation between CRS-R improvement (i.e., delta week 4 - baseline) and the time since injury using a Spearman correlation test. We also compared the treatment effect between etiologies (i.e., traumatic versus nontraumatic for active and sham intervention -4 groups) with a Kruskal-Wallis rank test. Each AE was collected from the Case Report Forms and expressed as a percentage of the total amount of sessions delivered. Adherence was expressed as a percentage of the total duration of planned stimulations (i.e., $6 \mathrm{~h}$ and $40 \mathrm{~min}$ ) as the device recorded the total stimulation duration for each initiated session. Finally, descriptive analyses at the group level were performed (CRS-R total score evolution expressed by median and interquartile range) and at the single subject level (CRS-R items recovered after the active and not after the sham treatment).

\section{Results}

As presented in Fig. 2, out of 86 eligible patients, 49 were not included because of one or several of the following reasons: no dedicated person could be found to apply tDCS daily, the patient lived too far away to enable proper assessments, they were modifications planned in the patient's treatment (either pharmacological or not) in the 6 upcoming months. Thirty-seven patients were assigned to receive both active and sham tDCS between June 2014 and January 2017. Ten patients were withdrawn from the study due to one of the abovementioned exclusion criteria (i.e., 5 unplanned treatment modifications, 3 infections, 1 severe adverse event and 1 transportation issue). These 10 patients did not differ from the 27 patients who completed the study in terms of age $(t=-0.624$; $\mathrm{p}=0.540)$, time since injury $(\mathrm{t}=0.836 ; \mathrm{p}=0.410)$ and baseline CRS-R $(\mathrm{t}=-1.470 ; \mathrm{p}=0.151)$.

Demographic data and CRS-R scores of the 27 patients who completed the protocol (mITT) are reported in Table 1. Out of these 27 patients, 5 patients did not receive at least 80\% (i.e., less than 16 sessions) of the active stimulation sessions and were thus excluded from the per protocol analysis ( $P P-n=22$ ).

All patients adequately tolerated all the tDCS sessions. Regarding the AE, skin redness was reported for 10 patients ( 5 during an active session and 5 during a sham session) and sleepiness for 3 patients ( 2 during an active and 1 during a sham session). An epileptic seizure was reported for one patient (day 4 of the first treatment period). This patient was immediately withdrawn from the study. Noteworthy, based on the device's report, that patient was receiving the sham intervention. In total, 13 mild AE were reported for a total amount of 946 sessions performed (1\%) and one severe AE (single episode of epileptic seizure) that was unrelated to tDCS (the patient was receiving sham stimulation). The sessions were performed by the family at home for 17 patients and by the nursing team for the remaining 10 patients. The adherence was $94 \pm 14 \%$ for the duration of stimulation (range: $48-130 \%-$ Fig. 3 ). Data were missing for one patient. As said above, 5 patients ( $18 \%$ of the study population) received less than $16(80 \%)$ active treatment sessions. Three of these patients were in a rehabilitation center and 2 were at home. On the other hand, 5 patients, who were at home, received more than 20 treatment sessions ( 3 active and 2 sham sessions). All the patients who presented an $\mathrm{AE}$ had a mean compliance of $96 \%$ (range: $80-130 \%$ ).

Regarding the changes in the CRS-R total score presented in Table 2, no carry-over effect was identified; mITT $(n=27)$ : $\mathrm{Z}=1.503, \mathrm{p}=0.132 ; \mathrm{PP}(\mathrm{n}=22): \mathrm{Z}=0.893 ; \mathrm{p}=0.372$. A moderate effect size was observed at the end of the 4 weeks of tDCS in favor of the active treatment; $\operatorname{mITT}(n=27)$ : effect size $=0.47 ; \mathrm{p}=0.053$; $P P(n=22)$ : effect size $=0.53 ; \mathrm{p}=0.043$. 


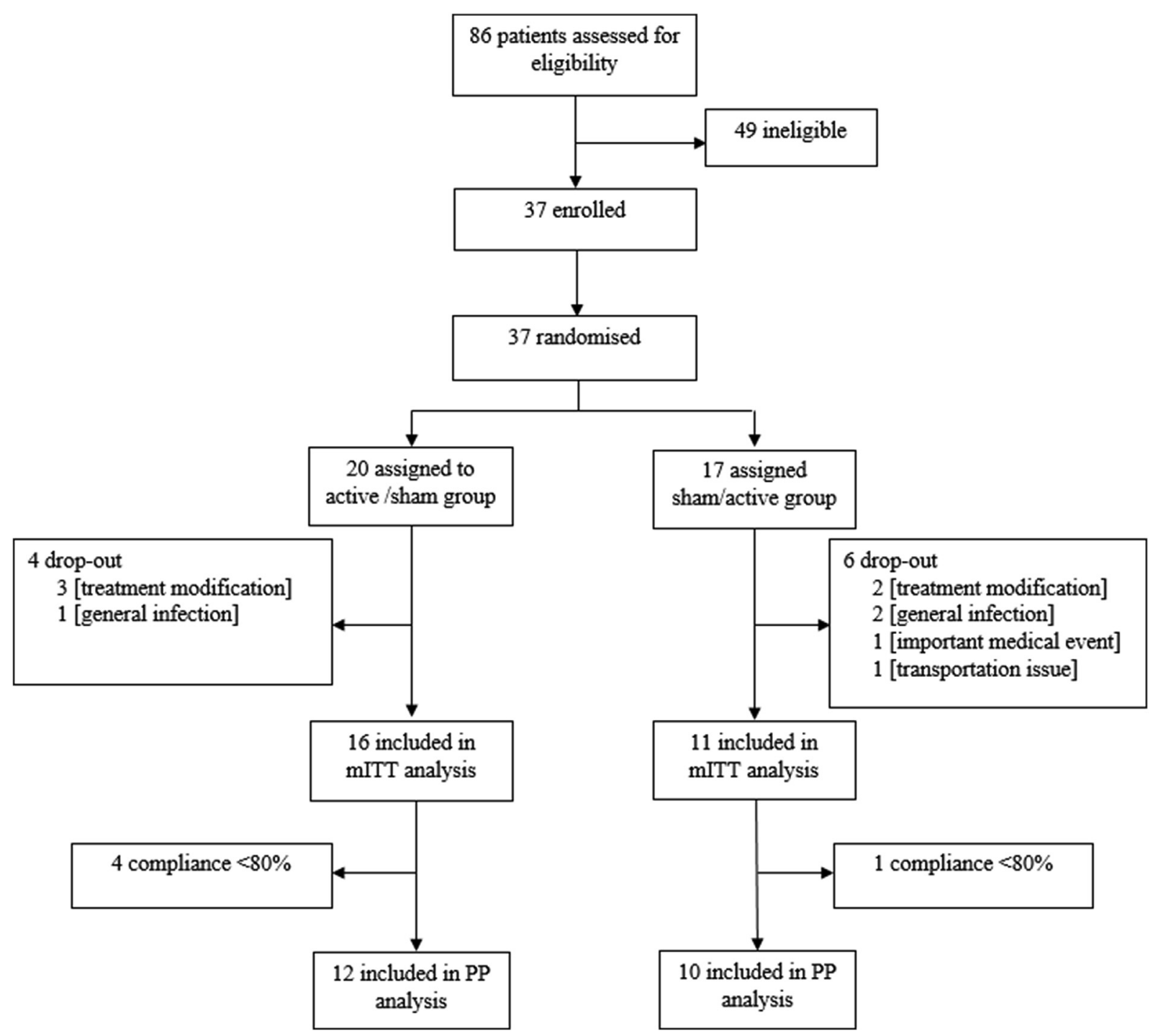

Fig. 2. CONSORT flow diagram.

No significant differences between groups' scores differences were found at 8 -week follow-up. A boxplot of the score variation is presented in Fig. 4.

We did not find any significant correlation between the time since injury and CRS-R improvement $(\rho=0.0213 ; p=0.878)$. Regarding the etiology, no difference between groups (active tDCStraumatic, active tDCS-non-traumatic, sham tDCS-traumatic, sham tDCS-non-traumatic) was identified $(\mathrm{H}=1.721 ; \mathrm{p}=0.6322)$.

As descriptive analyses, we identified a difference in the CRS-R individual items between the active and the sham groups after 4 weeks of tDCS for three items only, namely the object localization ( 3 patients in the active group presented this behavior after treatment, while no patient in the sham group), intentional communication ( 2 patients in the active group recovered this behavior after the active session, while no patient in the sham group) and object manipulation (present for 1 patient in the active group and none in the sham group).

\section{Discussion}

In this crossover, sham-controlled, randomized, double-blind study, we demonstrated the safety, the feasibility and the behavioral effects of 4 weeks of home-based tDCS in chronic MCS patients. This trial is the first tDCS study carried out in chronic patients with DOC in their daily environment.

Regarding our first outcome, we can conclude that applying 20 sessions of tDCS in patients with DOC appears to be safe, which is in line with previous observations $[8,21,22]$. However, one patient, who was under anti-epileptic medication (controlled epilepsy for 2 years), experienced a seizure. Since this patient only received sham tDCS, this severe AE is unrelated to tDCS and does not change the safety of repeated tDCS in patients with DOC. Indeed, the few AE reported (13/946 tDCS sessions - 1\%) refer to skin redness and sleepiness that both have been previously reported (no sign of pain or discomfort was reported) [23].

In addition, tDCS was properly delivered outside a research facility (second main outcome), as part of the daily cares that patients with DOC receive [24]. To monitor adherence in this remote setting, the device recorded the duration and number of completed stimulation sessions. At the group level, the overall adherence was excellent. When analyzing the individual adherence, we noticed that 5 patients received less than $80 \%$ of the treatment sessions. For 3 of them, tDCS was performed in addition to intensive rehabilitation interventions. It is possible that the schedule of these 
Demographic data, Coma Recovery Scale-Revised scores, adherence and MRI data. ${ }^{*}=$ patients who received less than $80 \%$ of the stimulations. CRS-R $=$ Coma Recovery Scale-Revised; Time $1=$ at baseline, Time $2=$ at 4 weeks (end of the treatment): Time $3=$ at 12 weeks ( 8 weeks after the end of the treatment). Adherence is expressed as part of the expected total time of stimulation (i.e., $6 \mathrm{~h}$ and $40 \mathrm{~min}$ ).

\begin{tabular}{|c|c|c|c|c|c|c|c|c|c|}
\hline \multirow[t]{2}{*}{ ID } & \multirow[t]{2}{*}{ Age (gender) } & \multirow[t]{2}{*}{ Etiology } & \multirow[t]{2}{*}{ Time since injury } & \multirow[t]{2}{*}{ Session } & \multicolumn{3}{|c|}{ CRS-R total score (subscores) } & \multirow[t]{2}{*}{ Adherence (\%) } & \multirow[t]{2}{*}{$\mathrm{CT} / \mathrm{MRI}$ main lesion } \\
\hline & & & & & Time 1 & Time 2 & Time 3 & & \\
\hline \multirow[t]{2}{*}{1} & $36(\mathrm{~F})$ & TBI & 12 years, 5 months & active & $10(3-3-2-1-0-1)$ & $15(3-4-4-1-1-2)$ & $10(1-3-2-2-1-1)$ & 100 & Right frontoparietal area, left frontal area, left temporal lobe \\
\hline & & & & sham & $10(1-3-2-2-1-1)$ & $6(1-1-2-1-0-1)$ & $8(3-1-2-1-0-1)$ & 95 & \\
\hline \multirow[t]{2}{*}{$2^{*}$} & 75(M) & ISCHEMIC STROKE & 11 years & active & $9(1-3-1-2-0-2)$ & $11(2-3-2-2-0-2)$ & $8(3-0-2-2-0-1)$ & 48 & Right frontal area, right basal nuclei \\
\hline & & & & sham & $8(3-0-2-2-0-1)$ & $8(1-2-2-1-0-2)$ & $5(0-1-0-2-0-2)$ & 100 & \\
\hline \multirow[t]{2}{*}{3} & $35(\mathrm{M})$ & TBI & 14 years & sham & $10(1-4-4-0-0-1)$ & $11(3-1-5-1-0-1)$ & $10(1-1-5-1-0-2)$ & 96 & Brainstem, frontal lobes, left thalamus \\
\hline & & & & active & $10(1-1-5-1-0-2)$ & $14(3-4-5-1-0-1)$ & $15(3-4-5-1-0-2)$ & 95 & \\
\hline \multirow[t]{2}{*}{4} & $35(\mathrm{~F})$ & TBI & 5 years, 3 months & sham & $13(3-5-1-1-1-2)$ & $15(3-3-5-1-1-2)$ & $13(3-3-4-1-0-2)$ & 95 & Mesencephalon, thalami, corpus callosum \\
\hline & & & & active & $13(3-3-4-1-0-2)$ & $15(3-3-5-1-1-2)$ & $16(3-5-4-1-1-2)$ & 95 & \\
\hline \multirow[t]{2}{*}{5} & $37(\mathrm{M})$ & CARDIAC ARREST & 13 years, 11 months & active & $9(1-3-2-1-0-2)$ & $5(1-0-1-1-0-2)$ & $9(1-3-1-2-0-2)$ & 105 & Thalami, hippocampus, basal nuclei \\
\hline & & & & sham & $9(1-3-1-2-0-2)$ & $6(1-0-1-2-0-2)$ & $9(1-3-1-2-0-2)$ & 95 & \\
\hline \multirow[t]{2}{*}{6} & $32(\mathrm{M})$ & TBI & 15 years, 4 months & active & $18(4-5-5-2-1-1)$ & $19(4-5-6-2-1-1)$ & $16(3-5-4-2-1-1)$ & 85 & Left thalamus, right frontal area \\
\hline & & & & sham & $16(3-5-4-2-1-1)$ & $19(4-5-6-2-1-1)$ & $18(4-5-5-2-1-1)$ & 100 & \\
\hline \multirow[t]{2}{*}{7} & $33(\mathrm{~F})$ & TBI & 15 years & sham & $14(3-3-5-1-0-2)$ & $14(3-3-5-1-0-2)$ & $12(3-3-3-1-0-2)$ & 100 & Bilateral frontal and frontobasal regions, right temporal lobe \\
\hline & & & & active & $12(3-3-3-1-0-2)$ & $12(3-3-3-1-0-2)$ & $13(3-4-3-1-0-2)$ & 93 & \\
\hline \multirow[t]{2}{*}{8} & 45(M) & TBI & 33 years, 5 months & sham & $7(0-3-2-1-0-1)$ & $8(1-3-2-1-0-1)$ & $6(1-1-2-1-0-1)$ & 100 & na \\
\hline & & & & active & $6(1-1-2-1-0-1)$ & $6(1-1-2-1-0-1)$ & $9(1-3-2-1-0-2)$ & 100 & \\
\hline 9 & $31(\mathrm{M})$ & TBI & 5 years, 2 months & sham & $5(1-0-1-2-0-1)$ & $4(0-0-1-2-0-1)$ & $5(0-0-1-2-0-2)$ & 95 & Brainstem, anterior left temporal pole, basal nuclei \\
\hline & & & & active & $5(0-0-1-2-0-2)$ & $8(3-0-1-2-0-2)$ & $8(3-0-1-2-0-2)$ & 100 & \\
\hline 10 & $63(\mathrm{~F})$ & ANEURYSM & 14 years & active & $14(3-1-5-3-0-2)$ & $14(3-1-5-3-1-1)$ & $15(3-1-5-3-1-2)$ & 100 & na \\
\hline & & & & sham & $15(3-1-5-3-1-2)$ & $11(3-0-5-2-0-1)$ & $12(3-0-5-3-0-1)$ & 98 & \\
\hline 11 & $45(\mathrm{M})$ & CARDIAC ARREST & 3 years, 10 months & sham & $10(2-2-2-2-0-2)$ & $6(1-1-2-0-0-2)$ & $9(2-1-2-2-0-2)$ & 100 & Basal nuclei \\
\hline & & & & active & $9(2-1-2-2-0-2)$ & $10(2-2-2-2-0-2)$ & $8(1-1-2-2-0-2)$ & 107 & \\
\hline 12 & $55(\mathrm{M})$ & ANEURYSM & 2 years, 11 months & active & $11(1-3-5-1-0-1)$ & $13(2-3-5-1-0-2)$ & $12(2-3-5-1-0-1)$ & 97 & Basal ganglia, left occipital lobe, right cerebellar hemisphere \\
\hline & & & & sham & $12(2-3-5-1-0-1)$ & $13(2-3-5-1-0-2)$ & $14(2-3-5-2-0-2)$ & 91 & \\
\hline 13 & $40(\mathrm{M})$ & CARDIAC ARREST & 10 years & sham & $6(1-1-1-1-0-2)$ & $8(1-3-1-1-0-2)$ & $6(1-1-1-1-0-2)$ & 105 & na \\
\hline & & & & active & $6(1-1-1-1-0-2)$ & $6(1-1-1-1-0-2)$ & $5(1-1-1-0-0-2)$ & 90 & \\
\hline 14 & $60(\mathrm{M})$ & ANEUVRYSM & 4 years, 1 months & active & $2(1-0-0-0-0-1)$ & $5(1-1-1-0-0-2)$ & $3(1-0-1-0-0-1)$ & 95 & Fronto-insular and temporal cortices \\
\hline & & & & sham & $3(1-0-1-0-0-1)$ & $3(1-0-1-0-0-1)$ & $5(1-0-1-2-0-1)$ & 89 & \\
\hline 15 & 57(M) & CARDIAC ARREST & 8 years, 8 months & active & $4(0-0-2-1-0-1)$ & $9(1-1-5-1-0-1)$ & $9(1-1-5-1-0-1)$ & 94 & Frontal, temporal and parietal lobes \\
\hline & & & & sham & $9(1-1-5-1-0-1)$ & $12(1-3-5-1-0-2)$ & $9(1-1-5-1-0-1)$ & 90 & \\
\hline $16^{*}$ & $46(\mathrm{~F})$ & CARDIAC ARREST & 1 years, 5 months & active & $3(1-1-0-0-0-1)$ & $4(1-1-0-1-0-1)$ & $5(1-1-0-2-0-1)$ & 54 & Basal ganglia, caudate nucleus, hippocampi \\
\hline & & & & sham & $5(1-1-0-2-0-1)$ & $4(1-1-0-1-0-1)$ & $8(2-2-1-2-0-1)$ & 63 & \\
\hline 17 & $33(\mathrm{M})$ & TBI & 8 years, 7 months & sham & $8(2-3-1-0-0-2)$ & $9(2-3-2-0-0-2)$ & $9(2-3-1-1-0-2)$ & 130 & Right frontal area, left temporal pole \\
\hline & & & & active & $9(2-3-1-1-0-2)$ & $12(2-4-2-2-0-2)$ & $10(2-3-2-1-0-2)$ & 101 & \\
\hline 18 & $55(\mathrm{M})$ & CARDIAC ARREST & 8 years, 2 months & active & $9(1-3-1-2-0-2)$ & $13(1-3-5-2-0-2)$ & $11(3-3-1-2-0-2)$ & 95 & Frontal and parietal lobes, left corpus callosum \\
\hline & & & & sham & $11(3-3-1-2-0-2)$ & $12(1-3-5-1-0-2)$ & $9(1-3-1-2-0-2)$ & 89 & \\
\hline $19^{*}$ & $48(\mathrm{~F})$ & ISCHEMIC STROKE & 10 months & active & $10(0-3-5-1-0-1)$ & $9(1-2-5-0-0-1)$ & $7(0-1-5-0-0-1)$ & 69 & Bilateral fronto-parieto-temporal areas and basal ganglia \\
\hline & & & & sham & $7(0-1-5-0-0-1)$ & $8(0-1-5-1-0-1)$ & $10(1-2-5-1-0-1)$ & 73 & \\
\hline 20 & $30(\mathrm{M})$ & CARDIAC ARREST & 3 years, 4 months & active & $9(1-4-0-2-0-2)$ & $7(2-1-0-2-0-2)$ & $9(2-4-0-1-0-2)$ & 80 & Left and right hemispheres \\
\hline & & & & sham & $9(2-4-0-1-0-2)$ & $9(2-4-0-1-0-2)$ & $10(3-4-0-1-0-2)$ & 100 & \\
\hline 21 & $38(\mathrm{M})$ & CARDIAC ARREST & 1 years, 2 months & active & $5(1-0-1-2-0-1)$ & $7(1-1-1-2-0-2)$ & $5(1-0-1-2-0-1)$ & 101 & Left frontal lobe \\
\hline & & & & sham & $5(1-0-1-2-0-1)$ & $5(1-0-1-1-0-2)$ & $8(1-1-2-2-0-2)$ & 100 & \\
\hline 22 & 23(M) & TBI & 2 years, 2 months & sham & $10(1-3-2-2-0-2)$ & $9(1-3-1-2-0-2)$ & $5(0-0-1-2-0-2)$ & 100 & Left thalamus, left frontal and temporal lobes, insula \\
\hline & & & & active & $5(0-0-1-2-0-2)$ & $7(1-0-2-2-0-2)$ & $8(1-1-2-2-0-2)$ & 95 & \\
\hline 23 & $70(\mathrm{~F})$ & ANOXIA & 4 years, 7 months & active & $15(3-3-5-2-0-2)$ & $14(3-3-4-2-0-2)$ & $15(3-4-4-2-0-2)$ & 125 & Left insula, left temporal lobe, basal ganglia, thalami \\
\hline & & & & sham & $15(3-4-4-2-0-2)$ & $14(3-3-5-2-0-1)$ & $16(3-4-6-2-0-1)$ & 95 & \\
\hline $24^{*}$ & $27(\mathrm{M})$ & TBI & 7 years, 11 months & sham & $8(1-3-2-1-0-1)$ & $9(1-3-2-1-0-2)$ & $12(3-3-2-2-0-2)$ & 95 & Frontal and temporal lobes, hippocampi \\
\hline & & & & active & $12(3-3-2-2-0-2)$ & $9(1-3-2-1-0-2)$ & $10(1-3-2-2-0-2)$ & 65 & \\
\hline 25 & $26(\mathrm{M})$ & TBI & 7 years, 4 months & sham & $7(0-3-2-1-0-1)$ & $12(2-3-5-1-0-1)$ & $11(1-3-5-1-0-1)$ & 100 & Cerebellum, right frontal lobe \\
\hline & & & & active & $11(1-3-5-1-0-1)$ & $14(3-3-5-1-0-2)$ & $12(3-4-2-1-0-2)$ & 100 & \\
\hline $26^{*}$ & $17(\mathrm{M})$ & TBI & 1 years, 9 months & active & $13(3-3-2-2-1-2)$ & $13(3-3-2-2-1-2)$ & $15(3-5-2-2-1-2)$ & MD & Fronto-temporal areas, basal ganglia, corpus callosum \\
\hline & & & & sham & $15(3-5-2-2-1-2)$ & $11(2-3-2-2-0-2)$ & $13(3-3-2-2-1-2)$ & MD & \\
\hline 27 & $42(\mathrm{~F})$ & CARDIAC ARREST & 1 years, 9 months & active & $7(1-1-1-2-0-2)$ & $9(1-3-1-2-0-2)$ & $8(2-1-1-2-0-2)$ & 81 & Basal ganglia, hippocampi \\
\hline & & & & sham & $8(2-1-1-2-0-2)$ & $13(3-5-1-2-0-2)$ & $6(2-0-1-1-0-2)$ & 100 & \\
\hline
\end{tabular}




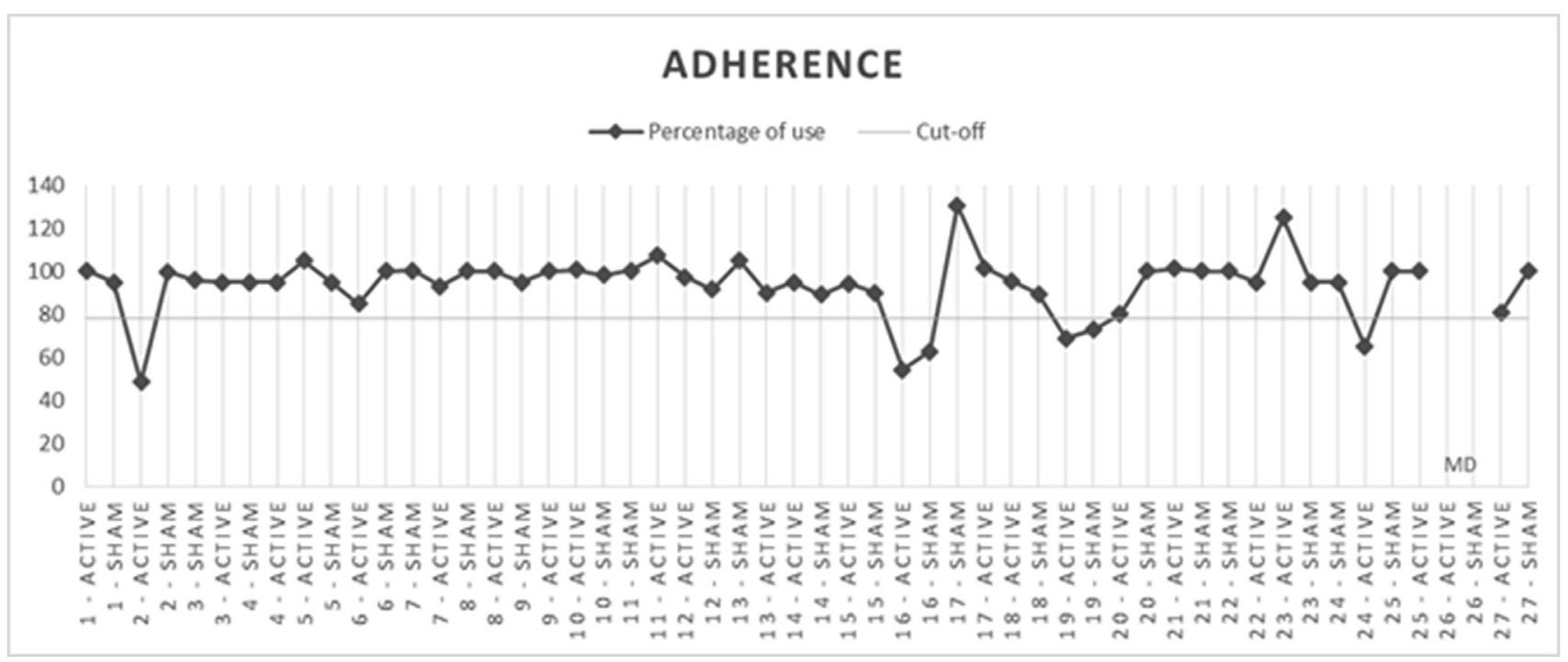

Fig. 3. Graph representing the adherence (i.e., percentage of use). $100 \%=6 \mathrm{~h} 40 \mathrm{~min}$; Cut-off $=80 \%$.

patients may have been burdensome and could explain why some sessions were missed. Missing sessions is indeed an important issue in tDCS studies which is not always properly tackled. We here adapted our analyses according to the proposed 80\% threshold [15]. Since this was a home-based protocol, an ongoing supervision was impossible and the adherence was highly variable as it is often the case with home-based studies [25]. In the future, combined interventions (e.g., tDCS during physical therapy) could be a valuable therapeutic approach to reduce the amount of missed sessions, even more since tDCS combined with other rehabilitation procedures can have greater effects [26-28]. At patients' homes, the data showed excellent adherence, meaning that the patients' relatives were able to use the device as indicated. Of course, home-based tDCS should always be monitored under close clinical supervision. To this end, Charvet and colleagues proposed 8 recommendations, which we followed in this study [29]. The same team applied these principles in a remotely-supervised tDCS randomized clinical trial (RCT) associated with cognitive training in patients with multiple sclerosis [30]. This study showed not only the feasibility of such a design in a large population but also the efficiency of combining tDCS with other rehabilitation procedures as abovementioned. Home-based tDCS thus appears to be a valuable therapeutic option, and may also improve feasibility and recruitment for future research in various populations. According to our results, the significant changes in CRS-R total scores at the group level suggest that 4 weeks of tDCS applied with the anode over the left DLPFC could enhance the level of consciousness by increasing responsiveness to external stimuli. The sample of the present study consisted mainly of MCS patients in an advanced chronic state; the median time since injury was 8 years. It is however conceivable to observe tDCS-related improvements at this stage since several cases of late recovery (i.e., years post injury) have been previously reported in patients with DOC [31-33]. MCS patients also have a better potential for late recovery than UWS patients [34]. One hypothesis supporting this relies on the potential white matter plasticity by axonal regrowth [35]. Another potential reason is the unmasking of cortical connections induced by non-invasive brain stimulation that results in neuroplasticity [36,37]. Therefore, it appears reasonable to assume that some chronic MCS may benefit from neuromodulation techniques such as tDCS. A critical issue of tDCS for patients with DOC is the duration of the effects. It is known that when applying one session of tDCS with the anode over the left DLPFC, the clinical improvements are small and do not last over time; while applying 5 daily sessions prolonged the effects for at least a week after the end of the stimulations [7]. In this study, we did not observe enduring clinical improvement at 8-week followup. The effects of tDCS were thus transient, suggesting that in chronic patients with MCS, tDCS should be maintained for the benefits to remain, or another strategy of delivering tDCS should be applied. This is not entirely in agreement with previous studies demonstrating positive tDCS-related long-term effects in depression or pain [22,38-40]. However, patients with MCS suffer from extensive brain damage which might explain the need for continuous neuromodulation to maintain neuroplastic changes.

At the individual level, we identified 6 patients (22\%) showing a new sign of consciousness at the end of the 20 active sessions that was not present after the sham sessions. This amount is lower than what we expected based on our previous studies (i.e., $43 \%$ after a single session and $56 \%$ after five daily sessions) [4,7]. However, as abovementioned, patients included in the present study were extremely chronic, up to 33 years post-injury as compared to these previous studies, and therefore this could have limited the potential

Table 2

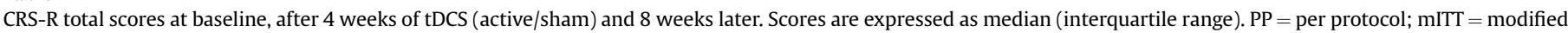
intention to treat.

\begin{tabular}{|c|c|c|c|c|c|c|}
\hline & \multicolumn{6}{|c|}{ CRS-R total score } \\
\hline & \multicolumn{3}{|c|}{ Active tDCS } & \multicolumn{3}{|l|}{ Sham tDCS } \\
\hline & Baseline & Week 4 & Week 12 & Baseline & Week 4 & Week 12 \\
\hline $\operatorname{mITT}(\mathrm{n}=27)$ & $9(6-12)$ & $10(7-14)$ & $9(8-13)$ & $9(7-12)$ & $9(6-12)$ & $9(6-12)$ \\
\hline $\mathrm{PP}(\mathrm{n}=22)$ & $9(6-11)$ & $11(7-14)$ & $10(8-14)$ & $10(7-12)$ & $10(6-13)$ & $9(6-12)$ \\
\hline
\end{tabular}




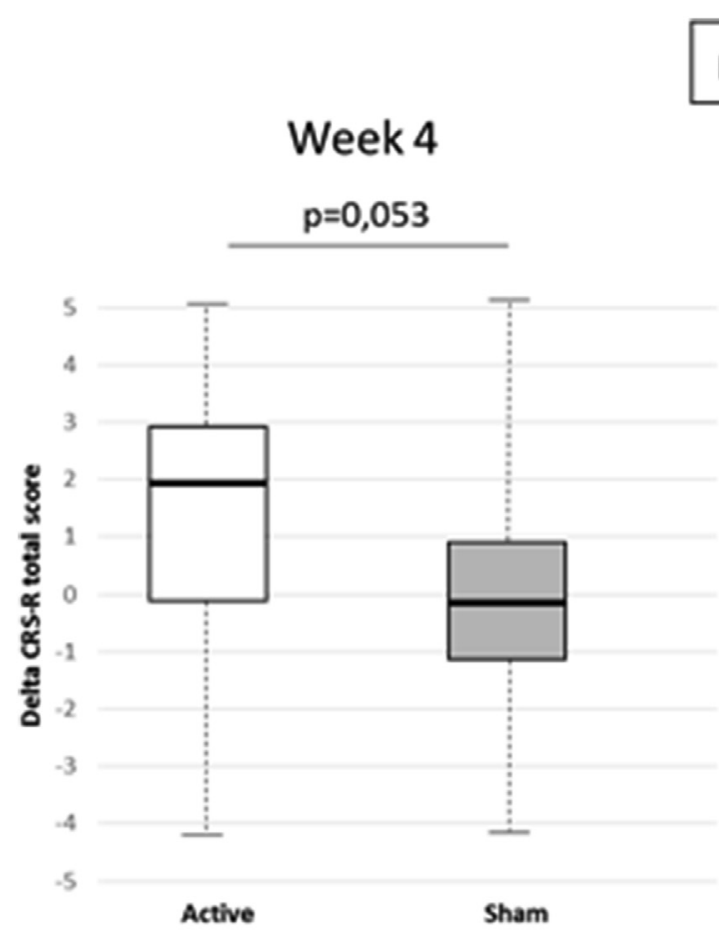

Effect size: 0.47

\section{$\operatorname{mITT}(n=27)$}

Week 4

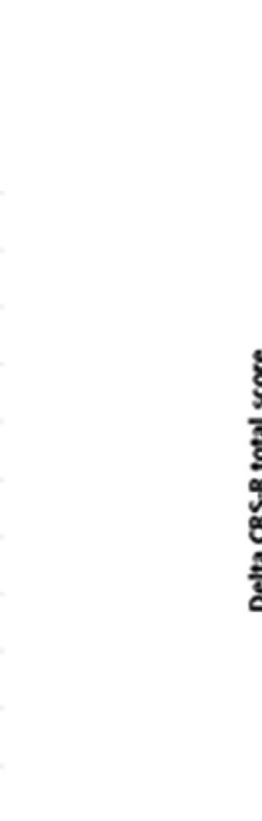

\section{Week 12}

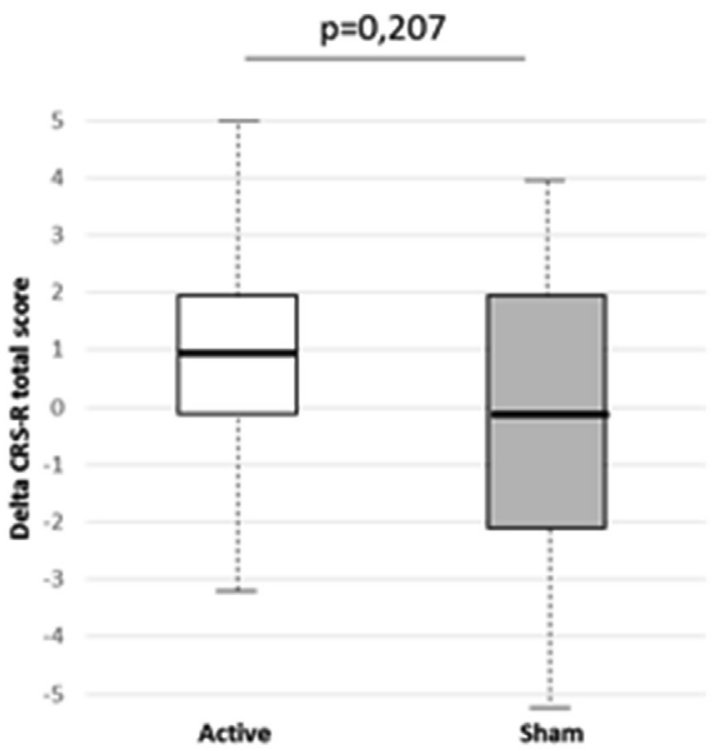

Effect size: 0.38



Effect size: 0.53

\section{PP $(n=22)$}


neuroplastic effects of tDCS. However, in view of the above results, tDCS may be used with other standard treatments, both in rehabilitation centers or at patients' home.

When comparing tDCS to other treatments available for patients in MCS such as amantadine, the effect size after 4 weeks of tDCS applied with the anode on the left DLPFC is higher ( 0.53 versus 0.24). However, the two populations and study designs were different and, therefore, we cannot claim that one treatment works better than the other [2].

This study encompasses some limitations that need to be taken into account before generalizing our results. No daily control on the tDCS application was done. However, our goal was to be as close as possible to clinical reality where caregivers and/or relatives provide a given treatment alone most of the time. Additionally, the person in charge of the stimulations was trained before starting the study and the investigators asked, at each visit, to show how the electrodes placement was done. AEs were only assessed by the family and/or the caregivers, and not directly collected by the investigators. This might raise concerns regarding patients' safety. However, considering our previous published tDCS studies performed on 104 patients did not report any serious adverse event (i.e., threatening the patient's health), the risk was minimal $[4,7,41]$. Regarding the assessments, patients were evaluated at baseline, after the 4-week stimulation period and at 8-week follow-up. Therefore, we were unable to evaluate the possible progression and regression of tDCS-related behavioral effects during other time windows. The duration of the whole protocol (i.e., 6 months) also led to a high number of patients being removed from the study (27\%) and thus to a small sample size. General infections and treatment modifications are the two main reasons for drop-outs, yet those are likely to happen during a 6-month period since this population is extremely vulnerable. Regarding the outcomes, we did not record neurophysiological measures such as EEG and our results only rely on behavioral assessments. Although, the neuronal correlate of a clinical response to tDCS has previously been investigated and highlighted the clinical response to tDCS requires structural and metabolic preservation (as measured by magnetic resonance imaging and positron emission tomography) in cortical and subcortical areas known to be involved in consciousness recovery [42]. However, since this is a clinical home-based RCT designed to assess clinical outcomes, we focused on behavioral measures rather than neurophysiological assessments. We can conclude that 20 sessions of home-based tDCS applied with the anode over the left DLPFC appear to be safe, feasible and to significantly improve patients' level of consciousness, for patients who adhere to the protocol. However, challenges for further studies with patients with DOC are multiple: 1 ) identifying tDCS responders beforehand by neuroimaging technology [42]; 2) implementing other biomarkers of tDCS responsiveness such as EEG which would also allow to better understand the electrophysiological mechanisms of tDCS in severely injured brains; 3 ) including more subjects to confirm and validate tDCS effectiveness; 4) increase the number of assessments both during treatment and at follow-up to assess more accurately the potential lasting effects of tDCS; and 5) explore combination therapies (i.e. tDCS and rehabilitation) to enhance the stimulation effects, as previously reported in other populations [26-28].

In conclusion, in this randomized controlled double blind clinical trial, we demonstrated that home-based tDCS was safe (1\% of mild AE) and can be used by patients' relatives at home or patients' caregivers in rehabilitation centers or nursing homes. In addition, when correctly applied, 4 weeks of tDCS in chronic (ranging from 10 months to 33 years post injury) MCS patients induced clinical improvements, even if moderate.

\section{Contributors}

AT and SL designed the protocol. GM and AT obtained and interpreted data and wrote the manuscript. ATO and AT analyzed the data. ATO, FF, NL, CM, SW and SL contributed to the writing of the manuscript. GM, AT and SL were the main investigators. All authors were involved in editing the paper and approved the final text.

\section{Disclosures}

GM, NL, ATO, FF, CM, SW, and AT declare no conflict of interests. $\mathrm{SL}$ is a consultant for Cefaly Technology.

\section{Study funding}

This research was supported by University and University Hospital of Liège (Belgium), Belgian National Funds for Scientific Research (FRS-FNRS), Human Brain Project (EU-H2020-fetflagshiphbp-sga1-ga720270), Luminous project (EU-H2020-fetopenga686764), Center-TBI project, James McDonnell Foundation, European Space Agency, Belspo, Fondazione Europea di Ricerca Biomedica, the BIAL Foundation, Wallonia Brussels Federation Concerted Research Action, Cefaly Technology and Mind Science Foundation. The funders had no role in study design, data collection and analysis, decision to publish, or preparation of the manuscript.

\section{References}

[1] Gosseries O, Charland-Verville V, Thonnard M, Bodart O, Laureys S, Demertzi A. Amantadine, apomorphine and zolpidem in the treatment of disorders of consciousness. Curr Pharmaceut Des 2014;20:4167-84.

[2] Giacino JT, Whyte J, Bagiella E, Kalmar K, Childs N, Khademi A, et al. Placebocontrolled trial of amantadine for severe traumatic brain injury. $\mathrm{N}$ Engl J Med 2012;366:819-26. https://doi.org/10.1056/NEJMoa1102609.

[3] Giacino JT, Ashwal S, Childs N, Cranford R, Jennett B, Katz DI, et al. The minimally conscious state: definition and diagnostic criteria. Neurology 2002;58:349-53.

[4] Thibaut A, Bruno M-A, Ledoux D, Demertzi A, Laureys S. tDCS in patients with disorders of consciousness Sham-controlled randomized double-blind study. Neurology 2014;82(13):1112-8. https://doi.org/10.1212 WNL.0000000000000260.

[5] Giacino JT, Kalmar K, Whyte J. The JFK Coma Recovery Scale-Revised: measurement characteristics and diagnostic utility. Arch Phys Med Rehabil 2004;85:2020-9.

[7] Thibaut A, Wannez S, Donneau A-F, Chatelle C, Gosseries O, Bruno M-A, et al. Controlled clinical trial of repeated prefrontal tDCS in patients with chronic minimally conscious state. Brain Inj 2017;31:1-9. https://doi.org/10.1080/ 02699052.2016.1274776.

[8] Estraneo A, Pascarella A, Moretta P, Masotta O, Fiorenza S, Chirico G, et al. Repeated transcranial direct current stimulation in prolonged disorders of consciousness: a double-blind cross-over study. J Neurol Sci 2017;375: 464-70. https://doi.org/10.1016/j.jns.2017.02.036.

[9] Angelakis E, Liouta E, Andreadis N, Korfias S, Ktonas P, Stranjalis G, et al. Transcranial direct current stimulation effects in disorders of consciousness. Arch Phys Med Rehabil 2014;95:283-9. https://doi.org/10.1016/ j.apmr.2013.09.002.

[10] Fregni F, Boggio PS, Nitsche M, Bermpohl F, Antal A, Feredoes E, et al. Anodal transcranial direct current stimulation of prefrontal cortex enhances working memory. Exp Brain Res 2005;166:23-30. https://doi.org/10.1007/ s00221-005-2334-6.

[11] Kang EK, Baek MJ, Kim S, Paik NJ. Non-invasive cortical stimulation improves post-stroke attention decline. Restor Neurol Neurosci 2009;27:645-50. https://doi.org/10.3233/RNN-2009-0514.

[12] Brunoni AR, Valiengo L, Baccaro A, Zanão TA, de Oliveira JF, Goulart A, et al. The sertraline vs electrical current therapy for treating depression clinical study. JAMA Psychiatry 2013;70:383. https://doi.org/10.1001/ 2013.jamapsychiatry.32.

[13] Castillo-Saavedra L, Gebodh N, Bikson M, Diaz-Cruz C, Brandao R, Coutinho L et al. Clinically effective treatment of fibromyalgia pain with high-definition transcranial direct current stimulation: phase II open-label dose optimization. J Pain 2016;17:14-26. https://doi.org/10.1016/j.jpain.2015.09.009.

[14] Wannez S, Heine L, Thonnard M, Gosseries O, Laureys S. The repetition of behavioral assessments in disorders of consciousness. Ann Neurol 2017;81(6): 883-9. 
[15] Thibaut A, O'Brien AT, Fregni F. Strategies for replacing non-invasive brain stimulation sessions: recommendations for designing neurostimulation clinical trials. Expet Rev Med Dev 2017;14:633-49. https://doi.org/10.1080/ 17434440.2017.1352470.

[16] Stagg CJ, Nitsche MA. Physiological basis of transcranial direct current stimulation. Neuroscientist 2011;17:37-53. https://doi.org/10.1177 1073858410386614.

[17] Herwig U, Satrapi P, Schonfeldt-Lecuona C. Using the international 10-20 EEG system for positioning of transcranial magnetic stimulation. Brain Topogr 2003; 16:95-9.

[18] Seel RT, Sherer M, Whyte J, Katz DI, Giacino JT, Rosenbaum AM, et al. Assessment scales for disorders of consciousness: evidence-based recommendations for clinical practice and research. Arch Phys Med Rehabil 2010;91:1795-813. https://doi.org/10.1016/j.apmr.2010.07.218.

[19] Raine TR, Harper CC, Rocca CH, Fischer R, Padian N, Klausner JD, et al. Direct access to emergency contraception through pharmacies and effect on unintended pregnancy and STIs: a randomized controlled trial. Jama 2005;293: 54-62. https://doi.org/10.1001/jama.293.1.54.

[20] Leuchter AF, Cook IA, Feifel D, Goethe JW, Husain M, Carpenter LL, et al. Efficacy and safety of low-field synchronized transcranial magnetic stimulation (sTMS) for treatment of major depression. Brain Stimul 2015;8:787-94. https://doi.org/10.1016/j.brs.2015.05.005.

[21] Sacco K, Galetto V, Dimitri D, Geda E, Perotti F, Zettin M, et al. Concomitant use of transcranial direct current stimulation and computer-assisted training for the rehabilitation of attention in traumatic brain injured patients: behavioral and neuroimaging results. Front Behav Neurosci 2016;10:57. https://doi.org/ 10.3389/fnbeh.2016.00057.

[22] Boggio PS, Rigonatti SP, Ribeiro RB, Myczkowski ML, Nitsche MA, PascualLeone $\mathrm{A}$, et al. A randomized, double-blind clinical trial on the efficacy of cortical direct current stimulation for the treatment of major depression. Int Neuropsychopharmacol 2008;11:249-54. https://doi.org/10.1017| S1461145707007833.

[23] Russo C, Souza Carneiro M, Bolognini N, Fregni F. Safety review of transcranial direct current stimulation in stroke. Neuromodulation 2017:20. 21-222.

[24] Saoût V, Ombredane MP, Mouillie JM, Marteau C, Mathé JF, Richard I. Patients in a permanent vegetative state or minimally conscious state in the Maine-etLoire county of France: a cross-sectional, descriptive study. Ann Phys Rehabil Med 2010;53:96-104. https://doi.org/10.1016/j.rehab.2010.01.002.

[25] Dunlay SM, Eveleth JM, Shah ND, McNallan SM, Roger VL. Medication adherence among community-dwelling patients with heart failure. Mayo Clin Proc 2011;86:273-81. https://doi.org/10.4065/mcp.2010.0732.

[26] Allman C, Amadi U, Winkler AM, Wilkins L, Filippini N, Kischka U, et al. Ipsilesional anodal tDCS enhances the functional benefits of rehabilitation in patients after stroke. Sci Transl Med 2016;8. https://doi.org/10.1126/scitranslmed.aad5651. 330re1-330re1.

[27] Nair DG, Renga V, Lindenberg R, Zhu L, Schlaug G. Optimizing recovery potential through simultaneous occupational therapy and non-invasive brainstimulation using tDCS. Restor Neurol Neurosci 2011;29:411-20. https:/ doi.org/10.3233/RNN-2011-0612.

[28] Dayan E, Cohen LG. Neuroplasticity subserving motor skill learning. Neuron 2011;72:443-54. https://doi.org/10.1016/j.neuron.2011.10.008.
[29] Charvet LE, Kasschau M, Datta A, Knotkova H, Michael C, Alonzo A, et al. Remotely-Supervised Transcranial Direct Current Stimulation ( Tdcs ) for Clinical Trials : Guidelines for Technology and Protocols, vol 9; 2015. p. 1-13. https://doi.org/10.3389/fnsys.2015.00026.

[30] Charvet L, Shaw M, Dobbs B, Frontario A, Sherman K, Bikson M, et al. Remotely supervised transcranial direct current stimulation increases the benefit of athome cognitive training in multiple sclerosis. Neuromodulation Technol Neural Interface 2017:2017. https://doi.org/10.1111/ner.12583.

[31] Schiff ND. Recovery of consciousness after brain injury: a mesocircuit hypothesis. Trends Neurosci 2010;33:1-9. https://doi.org/10.1016/ j.tins.2009.11.002.

[32] Sara M, Sacco S, Cipolla F, Onorati P, Scoppetta C, Albertini G, et al. An unexpected recovery from permanent vegetative state. Brain Inj 2007;21:101-3. https://doi.org/10.1080/02699050601151761.

[33] Estraneo A, Moretta P, Loreto V, Lanzillo B, Santoro L, Trojano L. Late recovery after traumatic, anoxic, or hemorrhagic long-lasting vegetative state. Neurology 2010;75:239-45.

https://doi.org/10.1212/ WNL.0b013e3181e8e8cc.

[34] Luaute J, Maucort-Boulch D, Tell L, Quelard F, Sarraf T, Iwaz J, et al. Long-term outcomes of chronic minimally conscious and vegetative states. Neurology 2010;75:246-52. https://doi.org/10.1212/WNL.0b013e3181e8e8df.

[35] Voss HU, Uluc AM, Dyke JP, Watts R, Kobylarz EJ, McCandliss BD, et al. Possible axonal regrowth in late recovery from the minimally conscious state. J Clin Invest 2006;116:2005-11. https://doi.org/10.1172/JCI27021.

[36] Fregni F, Pascual-Leone A. Hand motor recovery after stroke: tuning the orchestra to improve hand motor function. Cognit Behav Neurol 2006;19: 21-33.

[37] Simis M, Bravo GL, Boggio PS, Devido M, Gagliardi RJ, Fregni F. Transcranial Direct Current Stimulation in de novo Artistic Ability After Stroke. Neuromodulation Technol Neural Interface 2014;17:497-501. https://doi.org/ 10.1111/ner.12140.

[38] Ferrucci R, Bortolomasi M, Vergari M, Tadini L, Salvoro B, Giacopuzzi M, et al. Transcranial direct current stimulation in severe, drug-resistant major depression. J Affect Disord 2009:118.

[39] Brunoni AR, Ferrucci R, Bortolomasi M, Vergari M, Tadini L, Boggio PS, et al. Transcranial direct current stimulation (tDCS) in unipolar vs. bipolar depressive disorder. Prog Neuro Psychopharmacol Biol Psychiatr 2011;35:96-101. https://doi.org/10.1016/j.pnpbp.2010.09.010.

[40] Fregni F, Boggio PS, Lima MC, Ferreira MJ, Wagner T, Rigonatti SP, et al. A sham-controlled, phase II trial of transcranial direct current stimulation for the treatment of central pain in traumatic spinal cord injury. Pain 2006;122: 197-209. https://doi.org/10.1016/j.pain.2006.02.023.

[41] Huang W, Wannez S, Fregni F, Hu X, Jing S, Martens G, et al. Repeated stimulation of the posterior parietal cortex in patients in minimally conscious state: a sham-controlled randomized clinical trial. Brain Stimul 2017. https:// doi.org/10.1016/j.brs.2017.02.001.

[42] Thibaut A, Di Perri C, Chatelle C, Bruno MA, Bahri MA, Wannez S, et al. Clinical response to tDCS depends on residual brain metabolism and grey matter integrity in patients with minimally conscious state. Brain Stimul 2015;8(6): 1116-23. https://doi.org/10.1016/j.brs.2015.07.024. 